
\title{
25 Research Sourere \\ Evidence for ABL Amplification in Multiple Myeloma and Its Role in Treatment
}

\author{
He Huang \\ Wenzhou Medical University Second Affiliated Hospital \\ Wenjian Guo \\ Wenzhou Medical University Second Affiliated Hospital \\ Licai He \\ Wenzhou Medical University \\ Ronxin Yao \\ Wenzhou Medical University Second Affiliated Hospital \\ Ying Lin \\ Wenzhou Medical University Second Affiliated Hospital \\ Kang Yu \\ Wenzhou Medical University First Affiliated Hospital \\ Qian Li ( lisunqian1@163.com )
}

Department of Clinical Laboratory, The Second Affiliated Hospital and Yuying Children's Hospital of Wenzhou Medical University, Wenzhou, Zhejiang 325000, China

\section{Research}

Keywords: ABL amplification, Multiple myeloma, FISH, STI571

Posted Date: August 20th, 2020

DOI: https://doi.org/10.21203/rs.3.rs-48781/v1

License: (c) (1) This work is licensed under a Creative Commons Attribution 4.0 International License.

Read Full License 


\section{Abstract}

Background: The cytogenetic abnormalities are considered as initiating events in the pathogenesis of multiple myeloma (MM) and were assumed to be clinical significance. A number of defined cytogenetic lesions have been reported by genetic analysis techniques, while $A B L$ gene, known as the therapeutic target in chronic myelogenous leukemia (CML), its expression in myeloma has not been deeply explored.

Methods: We used publically available method FISH to analyze the chromosomal architecture, clinic features and overall survival of $101 \mathrm{MM}$ patient samples. Additionally, we examined ABL expression in MM cell lines (NCI-H929, LP-1 and U266) through FISH and western blot. After culturing with ABL kinase inhibitor STI571, we analyzed MM cell proliferation by CCK8 assay and detected ABL protein levels by western blot.

Results: Together with reported chromosomal abnormalities, we found ABL gene exhibited not as BCR$A B L$ fusion gene in CML, but its amplification was prevalent, 67 patients $(66.3 \%)$ had cytogenetic abnormalities with $A B L$ amplification. And the patients with $A B L$ gene amplification indicates no significance with clinical features, adverse cytogenetics (C-MYC amplification, IGH rearrangement, RB1 deletion, P53 deletion and 1q21 amplification) and overall survival comparing to patients with normal $A B L$ expression. Moreover, we revealed ABL amplification in MM cell lines (LP-1 and U266) by FISH, and $A B L$ protein was easy to detect in MM cell lines and some tumor cells. According to CML cells, the cytotoxicity of STI571 to MM cells was definitely limited.

Conclusions: Our study first discussed $A B L$ gene amplification in $M M$ cells, and we believe $A B L$ gene would potentially be a useful target in the treatment of combination strategy for MM with $A B L$ amplification in future.

\section{Introduction}

Multiple myeloma (MM) as one of the most common tumors of hematology, is characterized by malignant proliferation of plasma cells. Despite encouraging therapeutic advances, this disease remains an incurable disease due to complex genomic alterations, lower sensitivity to chemotherapy of MM cells in the bone marrow microenvironment and the emergence of drug resistance[1] Accumulating evidences have suggested that genotypic changes are found in $60 \%$ of patients at diagnosis by conventional chromosome analysis (CC) and in up to $90 \%$ of patients by fluorescence in situ hybridization (FISH) analysis. Some of these genetic abnormalities have been identified: rearrangement of the $14 \mathrm{q} 32(\mathrm{IgH})$ locus \c-myclcyclin D1\FGFR3 $\backslash$ cyclin D3, monoallelic deletions of chromosome 13, mutations of the Kras and N-ras genes, p53 monoallelic loss[2]. In addition, using DNA microarrays, a study compared the gene expression profiles of highly purified malignant plasma cells from nine patients with MM and eight myeloma cell lines to those of highly purified nonmalignant plasma cells (eight samples) obtained by in vitro differentiation of peripheral blood B cells. Overall there are two hundred and fifty genes were 
significantly up-regulated and 159 down-regulated in malignant plasma samples compared to normal plasma samples. For some of these confirmed genes, $A B L$ gene over-expressed in myeloma cells code for enzymes that could be a therapeutic target with specific drugs[3].

$A B L$ genes were first identified in the guise of a tumor gene in the Abelson murine lymphosarcoma virus, and ABL-family proteins comprise one of the best conserved branches of the tyrosine kinases. The product of the virally transduced oncogene ( $v-A B L)$, was determined to be an altered form of cellular $A B L$ (encoded by the C-ABL gene). ABL1 includes nuclear localization signals and a DNA binding domain through which it mediates DNA damage-repair functions, whereas ABL2 (also known as ABL related gene or Arg) has additional binding capacity for actin and for microtubules to enhance its cytoskeletal remodeling functions[4-6]. ABL genes are activated by chromosome translocations in various hematopoietic malignancies. Chronic myeloid leukemia (CML) is characterized in almost all cases by a $\mathrm{t}(9 ; 22)(\mathrm{q} 34 ; \mathrm{q} 11)$ translocation. Importantly, transformation by $A B L$ fusion proteins is inextricably tied to their tyrosine kinase activity, and targeted kinase inhibitor (Imatinib, also known as STI571or Gleevec) is therapeutically useful[ $[7,8]$.Wild-type $A B L$ is localized both in the nucleus and cytoplasm, and MM cells display high levels of nuclear $A B L$ in response to ongoing DNA damage and genomic instability. However most of its nuclear tumor suppressor functions are compromised because of the disruption of the ABLYAP1-p73 axis.Low YAP1 levels prevent nuclear ABL-induced apoptosis due to endogenous DNA damage,identifying a new synthetic-lethal strategy to selectively target cancer cells $[9,10]$.

DNA FISH techniques have become increasingly popular among genome biologists, now widely accepted as the primary methodology for the validation of Hi-C results. Thanks to the direct observation of the three-dimentional (3D) genome architecture in a manner that is complementary to chromosome conformation capture methods such as $\mathrm{Hi}-\mathrm{C}[11,12]$, as well as FISH probes produced, $\mathrm{FISH}$ is a versatile and expandable resource to study genome architecture, which can greatly facilitate the research and diagnostics of diseases.

Here, we first used FISH method to do chromosomal profiling and reported detailed ABL expression in $M M$ bone marrow samples and MM cell lines. The performance of $A B L$ gene amplification frequently appears even 3-fold or 4-fold in MM cells. Comparing to CML with BCR-ABL fusion gene, STI57 has poor anti-tumor effects in MM cells with $\mathrm{ABL}$ amplification through cell proliferation tests, western blot and FISH. Evidences have identified ABL is a selectively target of the synthetic-lethal strategy in MM. Information on $A B L$ regulatory mechanisms is being mined to provide new therapeutic strategies against hematopoietic malignancies not only BCR-ABL related types[13, 14]. Therefore, even ABL gene has been described in tumors as the response to DNA damage, we believed its role in the combination therapies.

\section{Methods And Regents}

\section{Patients}

We screened the database of the clinical cytogenetics laboratory at the Second Affliated Hospital and Yuying Children's Hospital of Wenzhou Medical University during the years June 2009 and July 2018. A 
total of $101 \mathrm{MM}$ including 11 relapsed patients were enrolled guided by the International Myeloma Working Group's criteria. Baseline data collected included the following: sex, age, M protein subtype in serum or urine, DS stage, serum levels of creatinine, Albumin, $\beta_{2}-M G, L D H$, and karyotype. All patients provided written informed consent for the use of their samples, and the study was approved by the ethics committee of the Second Affliated Hospital and Yuying Children's Hospital of Wenzhou Medical University.

\section{Cell lines, proteins and regents}

K562, NCl-H929, LP1, and U266 cell lines were obtained from the ATCC, and Cells were cultured in RPMI 1640 medium (Sigma-Aldrich, St. Louis, MO), supplemented with 10\% (vol/vol) heat-inactivated fetal bovine serum (FBS; Gibco BRL, Gaithersburg, $\mathrm{ML}$ ), in a humidified incubator at $37^{\circ} \mathrm{C}$ and $5 \% \mathrm{CO}_{2} / 95 \%$ air. Protein extracts of RPMI8226, NB4, U937, Kasumi-1, 293T, HCC1937, PC3, OVCAR-3 cells were gifted by Professor Wu (Department of Pathophysiology, Key Laboratory of Cell Differentiation and Apoptosis of Chinese Ministry of Education Shanghai Jiao-Tong University School of Medicine). Imatinib (STI571) was purchased from Selleck Chemicals (Houston, TX, USA) and prepared as a $1 \mathrm{mM}$ stock solution in DMSO at $-20^{\circ} \mathrm{C}$.

\section{Conventional karyotyping}

MM patients'bone marrow cells were cultivated for 24-48 hours without mitogen stimulation and harvested for chromosomal examination in a standard way. At least 20 metaphases R-banded by the Giemsa stain were examined, and the International System for Human Cytogenetic Nomenclature (2009) was used to describe chromosomal abnormalities.

\section{Fluorescence in situ hybridization}

We used the commercially available probes targeting C-MYC (8q24), RB1/D13S319 (13q14), P53/1q21 (17p13.1/1q21), IGH (14q32) and BCR/ABL (22q11/9q34) (GP Medical, Beijing, China) to detect 101 cases by inter/meta FISH. Interphase signals were evaluated in 200 nuclei of cells. Images were captured by a Nikon 80-A1 fluorescent microscope and analyzed with image analysis software Al.

\section{CCK8}

Cells were seeded into 96-well plates and incubated with various drug concentrations in triplicates for 48 h. Cell proliferation was assayed using a Cell Counting Kit (CCK8) (Dojindo Laboratories, Kumamoto, Japan) according to the manufacturer's instructions. Each experiment was conducted in triplicate and repeated three times.

\section{Western Blot}

Cells were harvested, washed with PBS and lysed with lysis buffer $(62.5 \mathrm{mM}$ Tris-HCl, $\mathrm{pH}$ 6.8, $100 \mathrm{mM}$ DTT, $2 \%$ SDS, $10 \%$ glycerol). Cell lysates were centrifuged at $20,000 \mathrm{~g}$ for 10 minutes at $4 \mathrm{uC}$, and proteins 
in the supernatants were quantified. Protein extracts were equally loaded to $6 \%$ to $15 \%$ SDSpolyacrylamide gels, electrophoresed, and transferred to nitrocellulose membrane (Amersham Bioscience, Buckinghamshire, United Kingdom). After blocking with 5\% nonfat milk in PBS for 2 hours at room temperature, the membranes were incubated with antibodies againstc-ABL was purchased from Santa Cruz Biotechnology (Santa Cruz, CA) overnight at $4^{\circ} \mathrm{C}$, followed by HRP-linked secondary antibody for 1 hour at room temperature. The signals were detected by chemiluminescence phototope-HRP kit (Millipore) according to manufacturer's instructions, and a-tubulin/ $\beta$-actin (Merck, Darmstadt, Germany) was probed as an internal control.

\section{Statistical analysis}

Depending on the distribution, the continuous data were presented as median (25th to 75th percentiles) or as mean \pm SD. Categorical data were presented as counts or proportions. The differences between the groups were assessed with the $\chi^{2}$ test or the Fisher's exact test for categorical data and the nonparametric Wilcoxon rank-sum test or a Student's t-test for continuous data. Univariate and multivariate logistic regression analyses were used to determine the relationship between $A B L$ amplification and normal $A B L$ expression groups. The analyses were performed using Empower $(R)$ (www.empowerstats.com, X\&Y Solutions, Inc. Boston MA) and R (http://www.R-project.org). And survival curves were constructed according to the Mantel-Cox method and compared using the log-rank test. A two-tailed value of $P<0.05$ was considered to indicate statistical significance.

\section{Results}

\section{ABL amplification is frequent in MM patients}

Between June 2009 and September 2018, 101 multiple myeloma patients (including 11 relapsed ones) with details were enrolled in the study, and we detected cytogenetic characteristic for each sample's bone marrow cells. The patients' baseline demographic and clinical features are summarized in Table 1. Among the MM patients, 67 patients (66.3\%) had cytogenetic abnormalities with ABL amplification identified by FISH. Here, no significant differences were observed between the ABL amplification and normal ABL expression groups with regard to sex, age, M protein, Durie-Salmon (DS) stage, LDH, creatinine, albumin, $\beta_{2}-\mathrm{MG}$ level, and karyotype (Table 1). In terms of cytogenetic features, we analyzed the incidences of C-MYC amplification, IGH rearrangement, P53 deletion, and 1q21 amplification in the $A B L$ amplification group were higher than those in the normal $A B L$ expression group, while the incidences of RB1/D13S319 deletion of in the ABL amplification group were lower than those in the normal $A B L$ expression group (56.7\% vs $64.8 \%$ ). However, no significant differences in those cytogenetic abnormalities (shown in Table 1) were observed between the two groups ( $P \otimes 0.05)$. In contrast, significant differences in karyotype performance were found between the two groups. Hyperdiploidy indicating better prognosis [15] was more likely to be found in $A B L$ amplification group, with the incidence was $32.8 \%$ (22/67), whereas the incidence in normal $A B L$ expression group was $5.9 \%(2 / 34)$. The incidence of normal karyotype in $A B L$ amplification group or $A B L$ normal expression group were $65.7 \%$ (44/67), and 
$76.5 \%$ (26/34), respectively. However, the incidence of hypodiploid implying poor prognosis[16] was $17.6 \%$ (6/34) for normal $A B L$ expression group and none in $A B L$ amplification group. In addition, we found only one sample exhibited polyploidy feature, coexisting with $A B L$ amplification, C-MYC amplification, IGH rearrangement, P53 deletion, and 1q21 amplification. We concluded ABL amplification was associated with genetic abnormalities, and myeloma cells were easy to find $A B L$ amplification in nucleus as the response of DNA injuries, particularly in cells withhyperdiploidy. Additionally, we further analyzed differences in overall survival (OS) between the two groups and 7 patients were lost to follow up ( 4 of $A B L$ amplification group and 3 of normal $A B L$ expression group). The results showed that the median survival of $A B L$ amplification group and normal $A B L$ expression group were 25 months and 34 months,but no significant difference was observed ( $P \otimes 0.05)$ (Figure 1).

\section{Chromosomal characteristics of MM cell lines}

To further assess the performance of FISH probes targeting small genomic loci in MM cell lines, we observed three human myeloma cell lines NCl-H929, U266 and LP-1 (they are characterized by hyperdiploidy karytype for 61-69 chromosomes and a variety of structural abnormalities) and CML cell line K562 with hyperdiploidy karytype and multi BCR-ABL fusion gene amplification. We detected C-MYC, RB1/D13S319, P53/1q21, IGH and BCR/ABL probes in NCI-H929, U266 and LP-1 cells. As shown in table 2 , we found $A B L$ amplification in $L P-1$ cells (three or four red signals of $A B L$ gene signifying $A B L$ gene amplification) and $U 266$ cells (four red signals of $A B L$ gene standing for of $A B L$ gene amplification), while $\mathrm{NCl}-\mathrm{H} 929$ plays normal $\mathrm{ABL}$ expression. According to Visualization of chromosomal territories by FISH chromosome-spotting probes, those high-risk genes were obtained based on Hi-C measurements. In NCl-H929 cells, C-MYC, IGH, D13S319/RB1 and 1q21 genes appeared triple amplification. In LP-1 cells, MYC amplified 6-fold, 1q21 amplified 8-10-fold and IGH rearranged. In U266 cells, C-MYC quadruple amplified, 1q21 amplified 6-fold, while variable region of IGH, P53 and D13S319/RB1 deleted. In Figure 2, C-MYC amplification, IGH rearrangement, P53 deletion, 1q21 amplification were confirmed in the metaphase or interphase FISH in these MM cell lines. In addition, RB1/D13S319 has differently chromosomes abnormalities, which amplifies in NCl-H929, normal in LP-1 and high ratio of deficiency in U266. Here, K562 cells were detected multi-BCR-ABL fusion gene amplification by FISH as the positive control.

\section{3. $\mathrm{ABL}$ expression in $\mathrm{MM}$ cell lines}

$A B L$ genes are found in all metazoans, including $A B L$ fusion genes can transform human fibroblasts in cultures, and enhanced $A B L$ signaling may contribute to epithelial cell malignancies, as well as to the invasive growth of breast cancer cells[17-19]. We then assessed ABL protein expression in both hematologic cells and solid tumors, and relatively easily found in most cells (Figure 3). The c-ABL protein expression could be identified in NCl-H929, LP-1, U266 and RPMI 8226 cell lines, while none of these cells exhibit BCR-ABL proteins. Even as described above, FISH was used to analyze ABL expression in nucleus of MM cell lines, and $\mathrm{NCl}-\mathrm{H} 929$ does not show ABL amplification. There is not only nuclear C-ABL, but also cytoplasmic c-ABL, therefore, we know ABL protein level can be not consist with FISH performance. 


\section{The cytotoxity of ABL inhibitor STI571to MM cells}

We took advantage of ABL kinase inhibitor STI571 (Imatinib) cultured with MM cells, and found that the STI571 inhibitory concentration necessary to obtain a $50 \%$ inhibition in MM cell proliferation $\left(\mathrm{IC}_{50}\right)$ is rather high $(\mathbb{1 0} \mu \mathrm{M})$ (Figure 4) compared to the BCR-ABL fusion gene expressing CML cell line K562 (0.5 $\mu \mathrm{M})$ (data not shown), which is consist with previous research[20]. And as shown in Figure 4, ABL protein expressions were dramatically blocked by the STI571 in MM cell lines. In addition, we used FISH to detect $A B L$ gene performance in cell nucleus, we just found the number of genetic loci indicating BCR-ABL fusion genes reduced in high concentration of STI571 cultured with K562 cells for long time (Figure 4C), and rarely impact ABL amplification in MM cells (data not shown). STI571 appears targeting BCR-ABL mainly in cytoplasm because it works by binding close to the ATP binding site, hardly interferes ABL gene expression in cell nucleus[21]. The results implied that $A B L$ kinase inhibitor can inhibit MM cells proliferation weakly comparing to CML cells, due to different mechanism of tumor cells development, and in the case of synergic effect on anti-myeloma, STI571 may work in some extent.

\section{Discussion}

$\mathrm{MM}$ is a genetically heterogeneous disease with a diverse clinical outcome and increasingly genetic abnormality will be explored in MM researches and diagnostics due to DNA technique development. Copy number alterations (CNAs), including whole chromosome and sub-chromosomal gains and losses, are common contributors of the pathogenesis and have demonstrated prognostic impact in MM [22]. In addition, diverse genomic landscapesingle nucleotide polymorphisme-array and next-generation sequencing (NGS) will extensively and deeply been profiled. At present, FISH a publically available resource enabling versatile DNA expression to study genome architecture, is a powerful method to study chromosomal organization in single cells. The novel method is reliable and can provide comprehensive profiling of disease-related unbalanced genetic aberrations with a short turn-around time, such as gene deletion, amplification, inversion and fusion gene detection. On the basis of these features, FISH could represent a valuable addition to diagnostic methods currently used for the genetic characterization of $\mathrm{MM}$, and provide the risk grades to indicate the prognosis of the disease, particular the patients with therapy including Bortezomib.

BCR-ABL fusion gene is involved in the Philadelphia chromosome in chronic myeloid leukemia, and rarely reported in myelomas. Even previous researches have confirmed over-expression of $A B L$ gene in malignant comparing to normal plasma cells with the Mann-Whitney nonparametric statistical test[3]. Here, we have shown the gene $A B L$ amplification was pervasive in MM patients and myeloma cell lines by FISH, and $A B L$ protein expression also was easy to detect in MM cells and other tumor cells. ABL gene amplification was more common in MM cells with hyperdiploid karyotypes, which did not occur alone and was often accompanied by known high-risk genetic abnormalities, including C-MYC amplification, IGH rearrangement, P53 deletion, and 1q21 amplification. Consisting with previous research [20], our results suggested TKI did not work in ABL amplification MM cells as its role in BCR-ABL positive cells. 
Although ABL kinase inhibitor STI571 revealed the weak cytotoxic effect to MM cells alone, it could be a therapeutic target with specific drugs.

This is the first article on the discussion the role of $A B L$ amplification in MM. Even we confirmed nucleus $A B L$ expression in MM patients, it is regret that we did not further sort cells by CD138 magnetic beads, which can show better validation of chromosome loci abnormality. This will be explored by a further study based on CD138 expressions immunophenotyping enrichment to assess the sample differences. As $A B L$ expression is prevalent in MM cells, a response to DNA damage, we found no significant clinical manifestation and survival differences between $A B L$ amplification groups and $A B L$ normal expression groups. And this result needs more samples to confirm. It is deficient we did not analyze the relationship of $A B L$ gene amplification and detailed prognosis (including progression free survival) for MM patients due to the different chemotherapies and poor or incomplete compliance. And the following work will be improved.

This is of particular interest since most tumor cells have a constitutive ABL activation, while TKI just plays apparently cytotoxic effect in few hematologic malignant tumors with BCR-ABL fusion gene, including CML, myelodysplastic syndrome (MDS)[23] and MM with BCR-ABL-positive[24]. However, combination of TKI and anti-IL- 6 antibodiesinduced a marked and significant inhibition on myeloma cells proliferation at low concentrations [3]. In MM, as well as other hematologic and solid malignancies, genomic instability, centrosome amplification and aneuploidy have been associated with the overexpression of Aurora kinases, a family of serine/threonine kinases that play essential and distinct roles in mitosis[25]. Accordingly, combined inhibition of Aurora and ABL kinases resulted in substantial cell death and tumor regression in MM related to NF-KB-inducing kinase (NIK)-c-ABL-STAT3 signalingcentered feedback loop[13]. Conversely, Zhen Caiet al indicated activation of c-ABL Kinase could potentiate the anti-myeloma drug Lenalidomide by promoting DDA1 protein recruitment to the CRL4 ubiquitin ligase. Furthermore, Panobinostat (HDAC inhibiotr) and Dexamethasone can enhance Lenalidomide-induced substrate degradation and cytotoxicity by activating c-ABL, providing a mechanism underlying their combination with Lenalidomide to treat MM[14]. In the past decade, three- or even four-drug regimens have been increasingly popular recommended for myeloma patients, $A B L$ kinase drugs can theoretically be applied. ABL includes nuclear localization signals and a DNA binding domain through which it mediates DNA damage-repair functions, whereas ABL in cytoplasm has additional binding capacity for actin and for microtubules to enhance its cytoskeletal remodeling functions. Several types of posttranslational modifications control ABL catalytic activity, subcellular localization, and stability, with consequences for both cytoplasmic and nuclear $A B L$ functions. Binding partners provide additional regulation of $A B L$ catalytic activity, substrate specificity, and downstream signaling [4]. Therefore, information on $A B L$ regulatory mechanisms is being further mined to provide new therapeutic strategies against hematopoietic malignancies with not only BCR-ABL fusion gene expression..

\section{Conclusions}


In conclusion, we used publically available and accepted technology FISH to study genome abnormalities in cells from MM patients'bone marrow, and first identified the gene $A B L$ amplification is frequent in MM. However, $A B L$ amplification did not have the relationship with clinical syndrome and outcome. Furthermore, we detected $A B L$ expression through FISH and western blot in vitro, and easy to find $A B L$ gene amplification and protein expression. Even the cell proliferation impacted by ABL kinase inhibitor STI571 is limited, we believe ABL gene would potentially be a useful target in the treatment of combination strategy for MM in future.

\section{List Of Abbreviations}

MM: Multiple myeloma

CML: Chronic myeloid leukemia

CC: Conventional chromosome analysis

FISH: Fluorescence in situ hybridization

DS: Durie-Salmon

OS: Overall survival

CNAs: Copy number alterations

NGS: Next-generation sequencing

MDS: Myelodysplastic syndrome

NIK: NF-KB-inducing kinase

\section{Declarations}

\section{Ethics approval and consent to participate}

This study was approved by the ethics committee of the Second Affliated Hospital and Yuying Children's Hospital of Wenzhou Medical University.

\section{Consent for publication}

Not applicable.

\section{Availability of data and materials}

The datasets supporting the conclusions of this article are included in this published article.

\section{Competing interests}

The authors declare no conflict of interest. 


\section{Funding}

This work was supported by Wenzhou Municipal Science and Technology Bureau (CN) (2019Y0658).

Authors' contributions

Conceptualization and design: Kang Yuand Qian Li. Development of methodology: He Huang, Wenjian Guo, Licai He and Qian Li. Acquisition of data: He Huang, Ying Lin and Qian Li. Analysis and interpretation of data: He Huang, Ronxin Yao and Qian Li. Writing and review of the manuscript: He Huang and Qian Li. Administrative, technical, or material support: He Huang, Wenjian Guo, Licai He and Qian Li. The authors read and approved the final manuscript.

Acknowledgements

We thank Professor Wu (Department of Pathophysiology, Key Laboratory of Cell Differentiation and Apoptosis of Chinese Ministry of Education Shanghai Jiao-Tong University School of Medicine) for kindly providing proteins.

\section{Reference}

1. Abramson HN: The Multiple Myeloma Drug Pipeline-2018: A Review of Small Molecules and Their Therapeutic Targets. Clinical lymphoma, myeloma \& leukemia 2018, 18(9):611-627.

2. Bergsagel PL, Kuehl WM: Chromosome translocations in multiple myeloma. Oncogene 2001, 20(40):5611-5622.

3. De Vos J, Thykjaer T, Tarte K, Ensslen M, Raynaud P, Requirand G, Pellet F, Pantesco V, Reme T, Jourdan $\mathrm{M}$ et al: Comparison of gene expression profiling between malignant and normal plasma cells with oligonucleotide arrays. Oncogene 2002, 21(44):6848-6857.

4. Colicelli J: ABL tyrosine kinases: evolution of function, regulation, and specificity. Science signaling 2010, 3(139):re6.

5. Witte $\mathrm{ON}$, Dasgupta A, Baltimore D: Abelson murine leukaemia virus protein is phosphorylated in vitro to form phosphotyrosine. Nature 1980, 283(5750):826-831.

6. Goff SP, Gilboa E, Witte ON, Baltimore D: Structure of the Abelson murine leukemia virus genome and the homologous cellular gene: studies with cloned viral DNA. Cell 1980, 22(3):777-785.

7. Schindler T, Bornmann W, Pellicena P, Miller WT, Clarkson B, Kuriyan J: Structural mechanism for STI571 inhibition of abelson tyrosine kinase. Science 2000, 289(5486):1938-1942.

8. Iqbal N: Imatinib: a breakthrough of targeted therapy in cancer. Chemotherapy research and practice 2014, 2014:357027. 
9. Cottini F, Hideshima T, Xu C, Sattler M, Dori M, Agnelli L, ten Hacken E, Bertilaccio MT, Antonini E, Neri A et al: Rescue of Hippo coactivator YAP1 triggers DNA damage-induced apoptosis in hematological cancers. Nature medicine 2014, 20(6):599-606.

10. Walters DK, Wu X, Tschumper RC, Arendt BK, Huddleston PM, Henderson KJ, Dispenzieri A, Jelinek DF: Evidence for ongoing DNA damage in multiple myeloma cells as revealed by constitutive phosphorylation of H2AX. Leukemia 2011, 25(8):1344-1353.

11. Lieberman-Aiden E, van Berkum NL, Williams L, Imakaev M, Ragoczy T, Telling A, Amit I, Lajoie BR, Sabo PJ, Dorschner MO et al: Comprehensive mapping of long-range interactions reveals folding principles of the human genome. Science 2009, 326(5950):289-293.

12. Gelali E, Girelli G, Matsumoto M, Wernersson E, Custodio J, Mota A, Schweitzer M, Ferenc K, Li X, Mirzazadeh $\mathrm{R}$ et al: iFISH is a publically available resource enabling versatile DNA FISH to study genome architecture. Nature communications 2019, 10(1):1636.

13. Mazzera L, Abeltino M, Lombardi G, Cantoni AM, Ria R, Ricca M, Saltarella I, Naponelli V, Rizzi FMA, Perris $\mathrm{R}$ et al: Functional interplay between NF-kappaB-inducing kinase and c-Abl kinases limits response to Aurora inhibitors in multiple myeloma. Haematologica 2019, 104(12):2465-2481.

14. Gao S, Geng C, Song T, Lin X, Liu J, Cai Z, Cang Y: Activation of c-Abl Kinase Potentiates the Antimyeloma Drug Lenalidomide by Promoting DDA1 Protein Recruitment to the CRL4 Ubiquitin Ligase. The Journal of biological chemistry 2017, 292(9):3683-3691.

15. Mei J, Zhai Y, Li H, Li F, Zhou X, Song P, Zhao Q, Yu Y, An Z, Wang L: Prognostic impact of hyperdiploidy in multiple myeloma patients with high-risk cytogenetics: a pilot study in China. Journal of cancer research and clinical oncology 2018, 144(11):2263-2273.

16. Van Wier S, Braggio E, Baker A, Ahmann G, Levy J, Carpten JD, Fonseca R: Hypodiploid multiple myeloma is characterized by more aggressive molecular markers than non-hyperdiploid multiple myeloma. Haematologica 2013, 98(10):1586-1592.

17. Lugo TG, Witte ON: The BCR-ABL oncogene transforms Rat-1 cells and cooperates with v-myc. Molecular and cellular biology 1989, 9(3):1263-1270.

18. Lin J, Arlinghaus R: Activated c-Abl tyrosine kinase in malignant solid tumors. Oncogene 2008, 27(32):4385-4391.

19. Srinivasan D, Plattner R: Activation of Abl tyrosine kinases promotes invasion of aggressive breast cancer cells. Cancer research 2006, 66(11):5648-5655.

20. Pandiella A, Carvajal-Vergara X, Tabera S, Mateo G, Gutierrez N, San Miguel JF: Imatinib mesylate (STI571) inhibits multiple myeloma cell proliferation and potentiates the effect of common antimyeloma agents. British journal of haematology 2003, 123(5):858-868. 
21. Vigneri P, Wang JY: Induction of apoptosis in chronic myelogenous leukemia cells through nuclear entrapment of BCR-ABL tyrosine kinase. Nature medicine 2001, 7(2):228-234.

22. Kosztolanyi S, Kiss R, Atanesyan L, Gango A, de Groot K, Steenkamer M, Jakso P, Matolcsy A, Kajtar B, Pajor $L$ et al: High-Throughput Copy Number Profiling by Digital Multiplex Ligation-Dependent Probe Amplification in Multiple Myeloma. The Journal of molecular diagnostics : JMD 2018, 20(6):777-788.

23. Doi S, Tashiro Y, Yoshinaga N, Kishimoto W, Hamada T, Ueda C, Moriguchi T, Kato K: [BCR-ABL1positive myelodysplastic syndrome with neutropenia and anemia treated successfully with imatinib mesylate]. [Rinsho ketsueki] The Japanese journal of clinical hematology 2020, 61(5):455-461.

24. Miki K, Obara N, Makishima K, Sakamoto T, Kusakabe M, Kato T, Kurita N, Nishikii H, Yokoyama Y, Sakata-Yanagimoto $\mathrm{M}$ et al: An Unprecedented Case of p190 BCR-ABL Chronic Myeloid Leukemia Diagnosed during Treatment for Multiple Myeloma: A Case Report and Review of the Literature. Case reports in hematology 2018, 2018:7863943.

25. Otto T, Sicinski P: Cell cycle proteins as promising targets in cancer therapy. Nature reviews Cancer 2017, 17(2):93-115.

\section{Tables}

Table 1 Comparison of the baseline features (101 MM cases 2009.6-2018.5) 


\begin{tabular}{|c|c|c|c|}
\hline Patient characteristics & $\begin{array}{l}\text { ABL amplification }(n=67) \\
\text { No. }(\%)\end{array}$ & $\begin{array}{l}\text { Normal ABL expression }(n=34) \\
\text { No. }(\%)\end{array}$ & $P$ \\
\hline \multicolumn{4}{|l|}{ Gender } \\
\hline Male & 48ه71.6区 & $20 \otimes 58.8 \rrbracket$ & 0.194 \\
\hline Female & $19 \rrbracket 28.4 \rrbracket$ & $14 \rrbracket 41.2 \rrbracket$ & \\
\hline \multicolumn{4}{|l|}{ Age(years) } \\
\hline & $36 \rrbracket 53.7 \rrbracket$ & $21 \otimes 61.8 \rrbracket$ & 0.292 \\
\hline & $31 \rrbracket 46.3 \rrbracket$ & $13 \rrbracket 38.2 \rrbracket$ & \\
\hline Median & 64 & 60 & \\
\hline \multicolumn{4}{|l|}{ M component } \\
\hline $\lg G$ & $44 \llbracket 65.7 \rrbracket$ & $18 \rrbracket 52.9 \rrbracket$ & 0.133 \\
\hline $\lg A$ & $20 \rrbracket 29.9 \rrbracket$ & $9 \rrbracket 26.5 \rrbracket$ & \\
\hline $\lg D$ & 0 & 0 & \\
\hline$\lambda$ & $1 \otimes 1.5 \rrbracket$ & $3 \rrbracket 8.8 \rrbracket$ & \\
\hline K & $2 \varangle 3.0 \rrbracket$ & $4 \otimes 11.8 \rrbracket$ & \\
\hline \multicolumn{4}{|l|}{ DS stage } \\
\hline 1 & $7 \rrbracket 10.4 \rrbracket$ & $1 \rrbracket 2.9 \rrbracket$ & 0.476 \\
\hline$\|$ & $4 \llbracket 6.0 \rrbracket$ & $2 \varangle 5.9 \rrbracket$ & \\
\hline III & $56 \rrbracket 83.6 \rrbracket$ & $31 \otimes 91.2 \rrbracket$ & \\
\hline \multicolumn{4}{|l|}{ Creatinine $(\mu \mathrm{mol} / \mathrm{L})$} \\
\hline & $55 \rrbracket 82.1 \rrbracket$ & $33 \otimes 97.1 \rrbracket$ & 0.055 \\
\hline & $12 \bowtie 17.9 \rrbracket$ & $1 \otimes 2.9 \rrbracket$ & \\
\hline \multicolumn{4}{|l|}{ Albumin $(\mathrm{g} / \mathrm{L})$} \\
\hline & $5 \bigotimes 7.5 \rrbracket$ & $7 \rrbracket 20.6 \rrbracket$ & 0.099 \\
\hline & $62 \varangle 92.5 \rrbracket$ & $27 \rrbracket 79.4 \rrbracket$ & \\
\hline \multicolumn{4}{|l|}{$\beta_{2}-M G(m g / L)$} \\
\hline & $19 \rrbracket 28.4 \rrbracket$ & 16ه47.1》 & 0.228 \\
\hline \multirow[t]{2}{*}{$3.5-5.5$} & $26 \rrbracket 38.8 \rrbracket$ & $9 \llbracket 26.5 \rrbracket$ & \\
\hline & $22 \rrbracket 32.8 \rrbracket$ & $9 \rrbracket 26.5 \rrbracket$ & \\
\hline LDH (U/L) & & & \\
\hline
\end{tabular}




\begin{tabular}{|c|c|c|c|}
\hline & 49凶73.1》 & $27 \bigotimes 79.4 \rrbracket$ & 0.475 \\
\hline & $18 \bowtie 26.9 \rrbracket$ & $7 \rrbracket 20.6 \rrbracket$ & \\
\hline Karyotype & & & \\
\hline Hyperdiploidy & $22 \bowtie 32.8 \rrbracket$ & $2 \varangle 5.9 \rrbracket$ & $<0.001$ \\
\hline Hypodiploid & 0 & 6冈17.6凹 & \\
\hline Polyploidy & $1 \rrbracket 1.50 \rrbracket$ & 0 & \\
\hline Normal karyotype & 44囚65.7】 & $26 \rrbracket 76.5 \rrbracket$ & \\
\hline $\begin{array}{l}\text { Coexistent adverse } \\
\text { cytogenetics }\end{array}$ & & & \\
\hline C-MYC amplification & $31(46.3)$ & 10邓29.4】 & 0.103 \\
\hline IGH rearrangement & $45(67.2)$ & $20(58.8)$ & 0.408 \\
\hline RB1/D13s319d & $38(56.7)$ & $22(64.7)$ & 0.528 \\
\hline 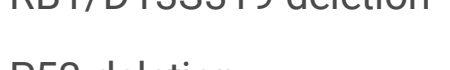 & $5(14.9)$ & $2(5.9)$ & 0.768 \\
\hline $1 q 21$ amplification & $34(50.7)$ & $15(44.1)$ & 0.440 \\
\hline
\end{tabular}

Table 2 Multiple myeloma cell lines genetic profiling by FISH

\begin{tabular}{|lllll|}
\hline Karyotype & NCl-H929 & LP-1 & U266 & K562 \\
\hline BCR/ABL & $2 \mathrm{G} 20$ & $3 \mathrm{G} 30=70 \% / 4 \mathrm{G} 40=30 \%$ & $4 \mathrm{G} 40=25 \%$ & Multiple F \\
\hline C-MYC & $3 \mathrm{~F}$ & $6 \mathrm{~F}$ & $4 \mathrm{~F}=27 \%$ & \\
\hline IGH & $3 \mathrm{~F}$ & $2 \mathrm{~F} 6 \mathrm{G} 20$ & $2 \mathrm{~F} 2 \mathrm{O}=21 \%, \mathrm{FO}=79 \%$ & \\
\hline D13S319/RB1 & $3 \mathrm{G} 30$ & $2 \mathrm{G} 20$ & $2 \mathrm{G} 2 \mathrm{O}=19 \%, \mathrm{GO}=81 \%$ & \\
\hline P53/1q21 & $2 \mathrm{G} 30$ & $3 \mathrm{G} 8-100$ & $2 \mathrm{G} 60=26 \%, \mathrm{G} 20=74 \%$ & \\
\hline
\end{tabular}

F: Fusion, G: Green, O: Orange/Red, 2G20: Normal

\section{Figures}




\section{OS}

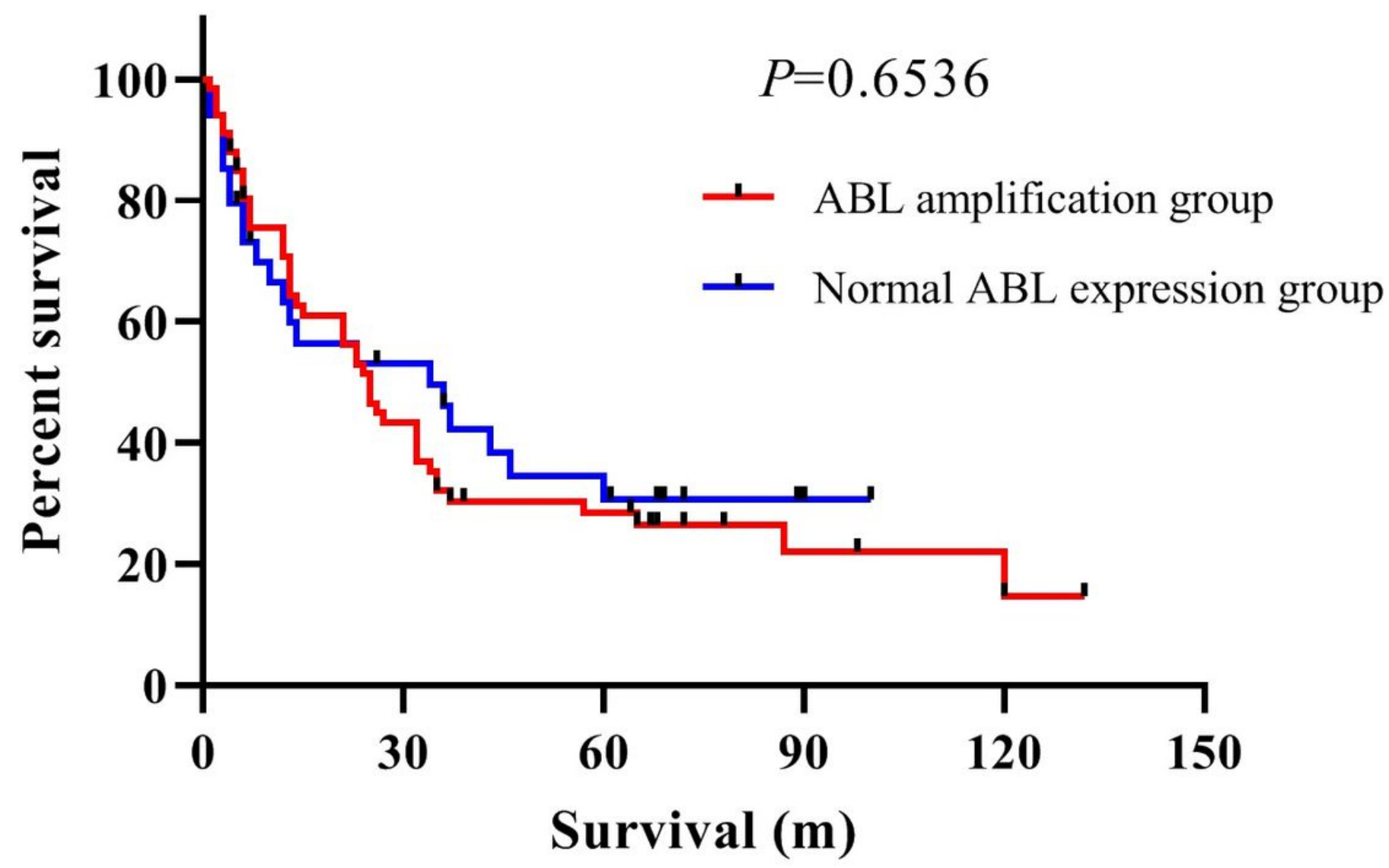

Figure 1

Survival analyses of MM patients. Mantel-Cox curves demonstrated the OS of the ABL amplification patients $(n=63)$ compared with the normal ABL expression patients $(n=31)$. 
A

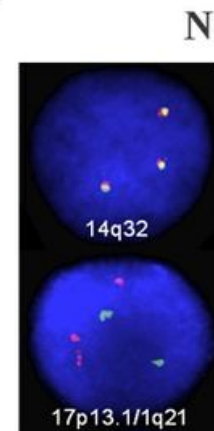

NCI-H929

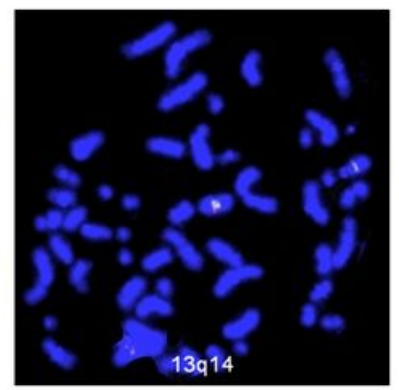

B

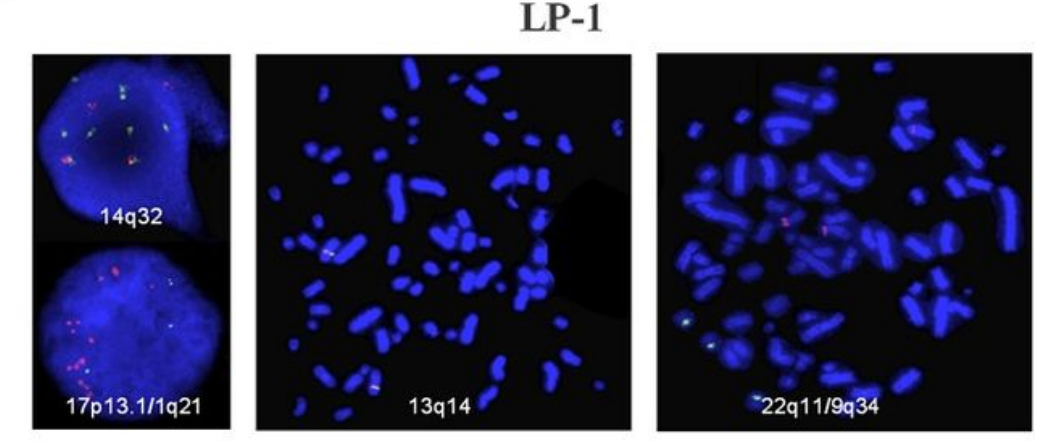

C

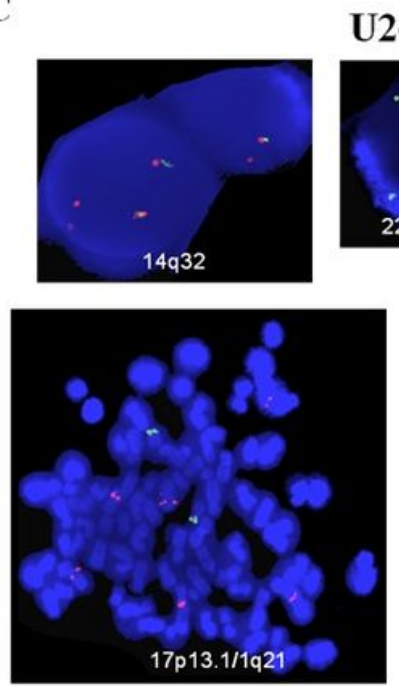

U266
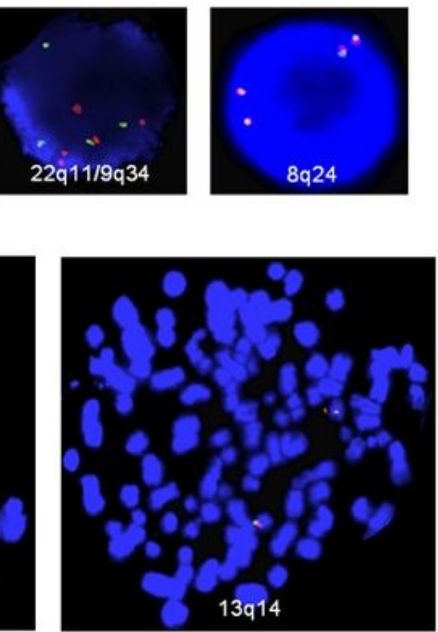

D

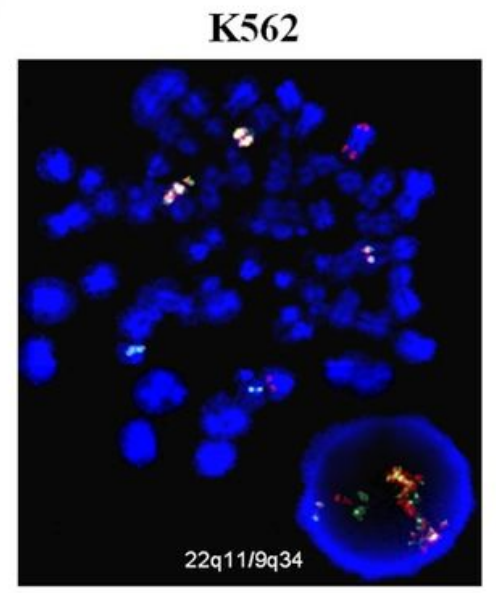

Figure 2

Copy number abnormalities were detected by FISH in MM cell lines. GLP IGH dual color breakpoint probe (located at 14q32), P53/1q21 probe (located at 17p13.1/1q21), D13S319/RB1 probe (located at 13q14), GLP C-MYC dual color breakpoint probe (located at 8q24), and GLP BCR-ABL dual color fusion probe (located at 22q11/9q34). A. NCl-H929: In interphase FISH, the picture displayed three fusion signals indicating IGH gene amplification, two green signals and three red signals signifying P53 gene normal and 1q21 amplifying. In metaphase FISH, the picture displayed three green and three red signals indicating D13S319■RB1 gene amplification. B. LP1: In interphase FISH, the picture displayed two fusion, six green and two red signals indicating IGH gene rearrangement and amplification, three green signals and ten red signals signifying P53 gene and 1q21 amplifying. In metaphase FISH, the picture displayed two green and two red signals indicating D13S3190RB1 gene normal, but it can show chromosome 13 transformed into derivative chromosome 13. And the picture displayed three green and three red signals indicating BCR $\square A B L$ gene amplification. C. U266: In interphase FISH, the picture displayed in diploid cells, one fusion and one red signals indicating IGH gene rearrangement and IGH variant region deletion. In tetraploid cells, two fusion and two red signals, indicated IGH gene rearrangement and IGH variant region deletion. And the picture displayed four green and four red signals indicating BCR $\square A B L$ gene amplification, four fusion signals indicating MYC gene amplification. In metaphase FISH, the picture 
showed two green signals and six red signals signifying P53 gene deletion and 1q21 amplifying, two green and two red signals indicating D13S319RB1 gene normal. D. K562: In metaphase FISH, the picture displayed two idic $(\mathrm{Ph})$, another one fusion signal $\$ three red $\mathrm{ABL}$ and two $\mathrm{BCR}$ genes. And in interphase FISH, the picture displayed multiple fusion genes and three red $A B L$ and two BCR genes.
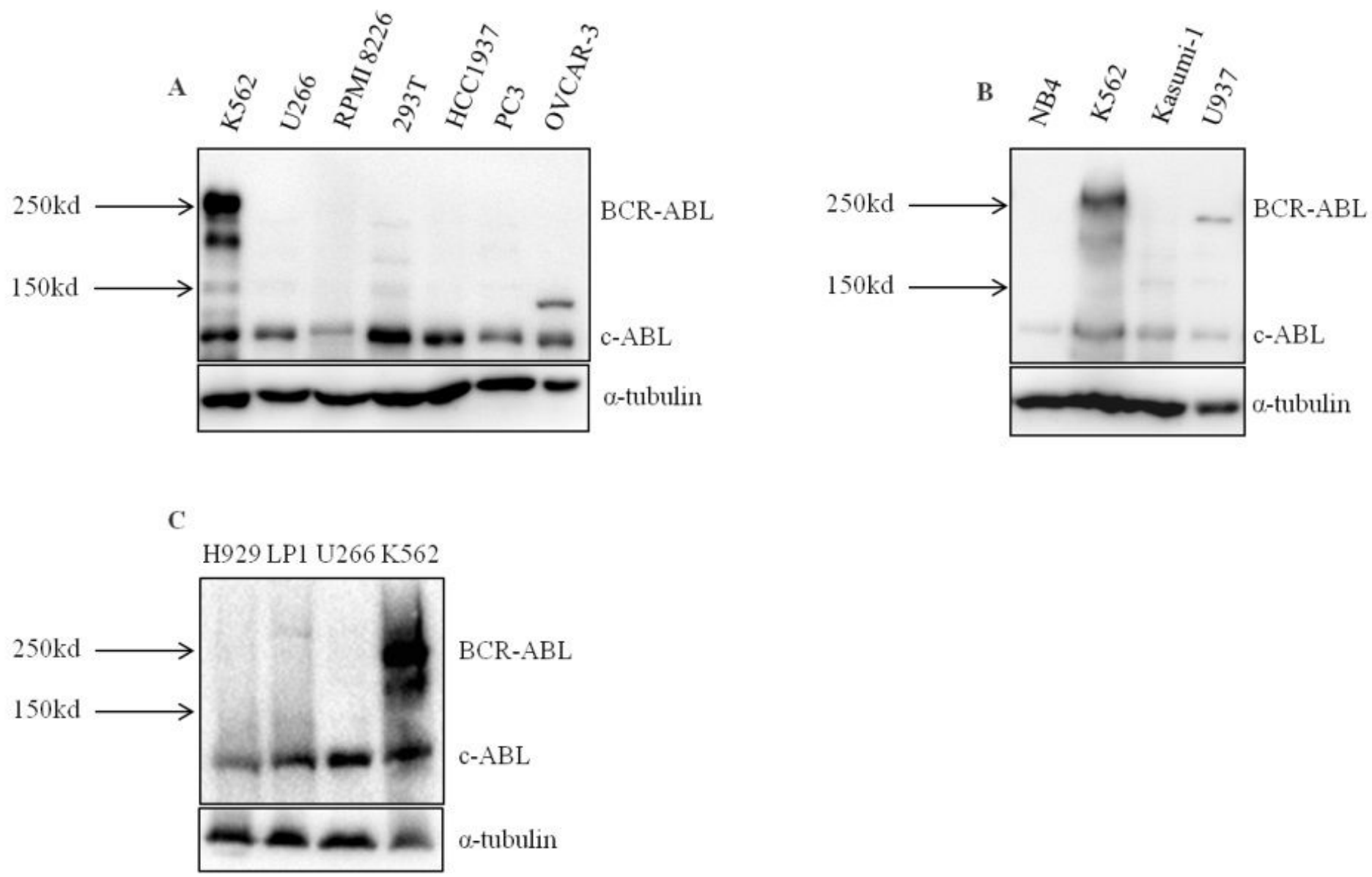

\section{Figure 3}

$A B L$ protein expression measured by western blot. 


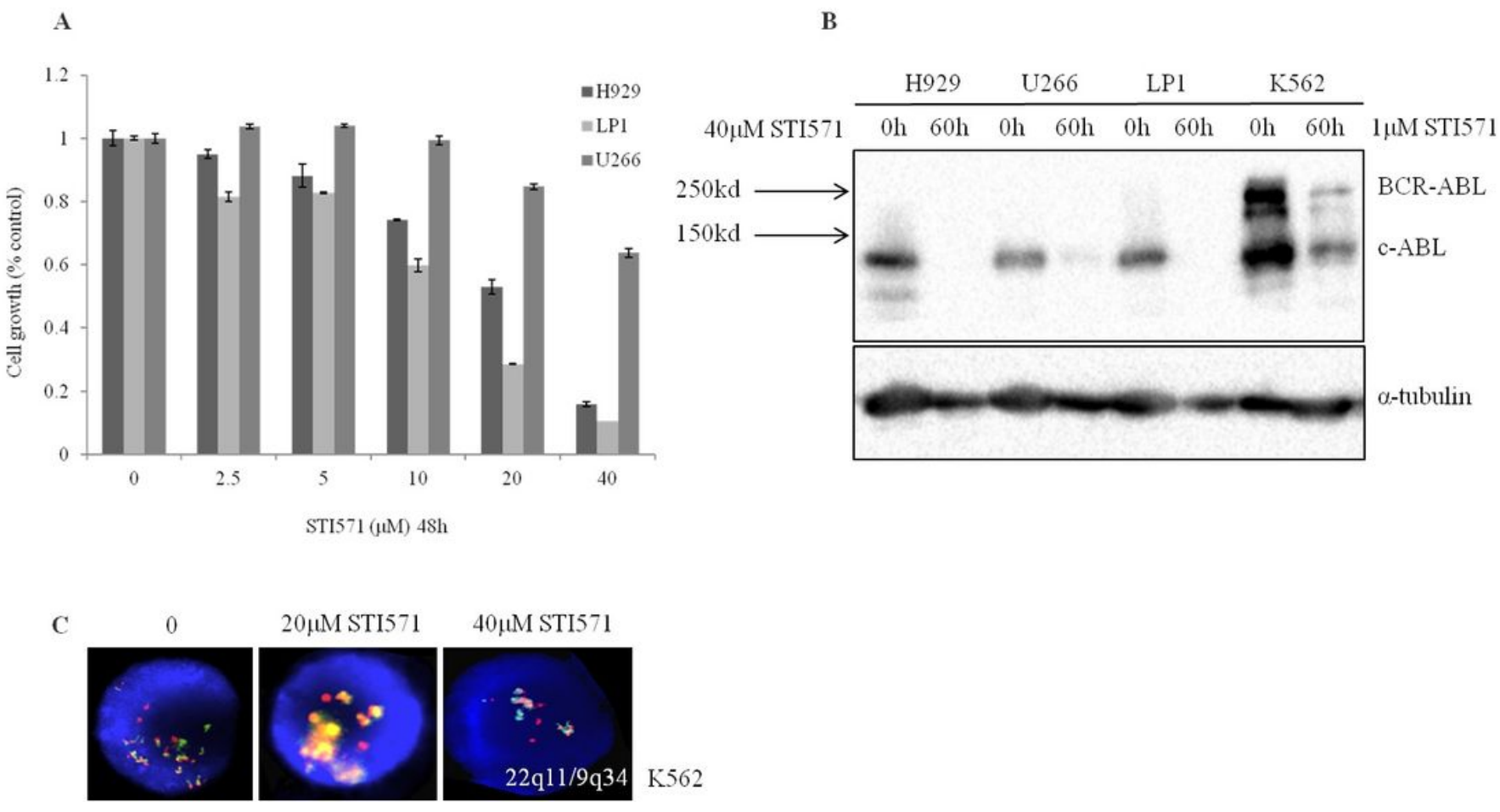

Figure 4

Effect of STI571 in MM cells. A. MM cell lines (NCl-H929, LP1 and U266) were cultured with concentrations of STI571 for $48 \mathrm{~h}$ and the proliferation of cells was assayed by CCK8. All values represent means $\pm S D$ of three independent experiments, each performed in triplicate. B. Western blot analysis of BCR-ABL and c-ABL protein expressions in MM together with CML cell (K562) lines treated with STI571 for $48 \mathrm{~h}$. C. The gene changes impacted by STI571 measured by FISH analysis with GLP BCR-ABL dual color fusion probe (located at 22q11/9q34).In interphase FISH, the picture displayed multiple fusion genes, three red $A B L$ and two BCR genes. BCR-ABL fusion genes exhibited no change and slightly reduced when add $20 \mu \mathrm{M}$ and $40 \mu \mathrm{M}$ STI571 for $60 \mathrm{~h}$, respectively. 